Schreiter, K.; Müller, S.; Luckner, R.; Manzey, D.

\title{
Enhancing manual flight precision and reducing pilot workload using a new manual control augmentation system for energy angle
}

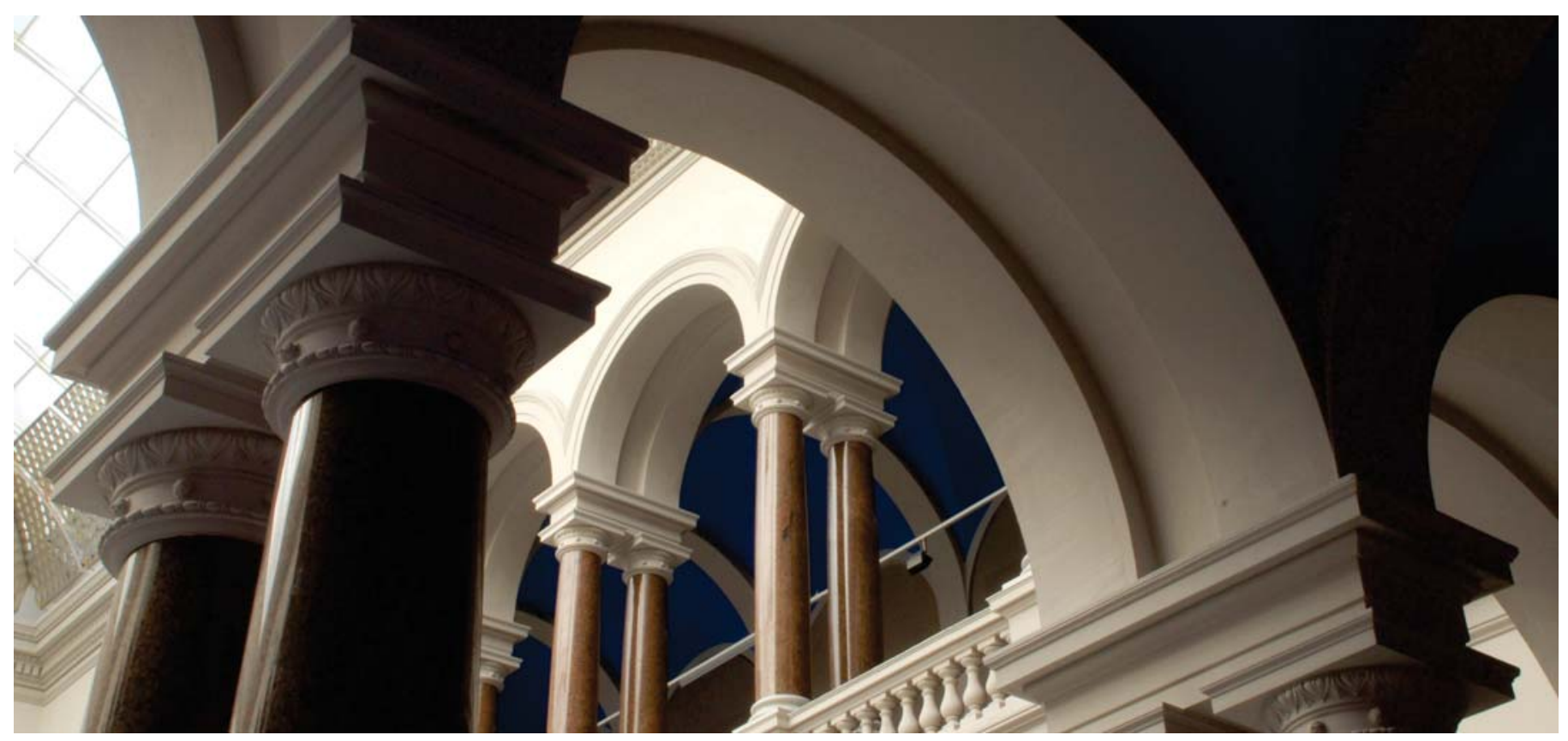

Schreiter, K., Müller, S., Luckner, R., \& Manzey, D. (2017). Enhancing Manual Flight Precision and Reducing Pilot Workload Using a New Manual Control Augmentation System for Energy Angle. In AIAA Guidance, Navigation, and Control Conference. American Institute of Aeronautics and Astronautics. https://doi.org/10.2514/6.2017-1026 


\title{
Enhancing Manual Flight Precision and Reducing Pilot Workload Using a New Manual Control Augmentation System for Energy Angle
}

\author{
K. Schreiter*, S. Müller ${ }^{\dagger}$, R. Luckner ${ }^{\ddagger}$ and D. Manzey ${ }^{\S}$ \\ Technische Universität Berlin, 10587 Berlin, Germany
}

\begin{abstract}
With rising demands on flight precision and more complex flight trajectories, pilots' workload during manual flight is increasing. This is especially the case for thrust and spoiler control during approach and landing. The presented nxControl system enables pilots to manually control the longitudinal load factor $n_{x}$ instead of engine parameters and spoiler deflections. This load factor is equivalent to total energy angle and is directly influenced by engine thrust and aerodynamic drag. The nxController complements existing control augmentation systems such as the fly-by-wire control laws of today's commercial airliners. It aims at higher precision with lower workload during manual flight. The controller input can be set and monitored by an adapted human-machine interface consisting of a thrustlever-like inceptor and additional display elements to enhance energy awareness. This paper presents the $\mathrm{nxControl} \mathrm{system} \mathrm{with} \mathrm{focus} \mathrm{on} \mathrm{the} \mathrm{command} \mathrm{control} \mathrm{system} \mathrm{and} \mathrm{an} \mathrm{evaluation}$ study with 24 airline pilots in a research flight simulator. The task was a demanding and steep approach with required navigation performance RNP 0.1 in a mountainous area. The results show higher precision and lower workload with the nxControl system despite minimal amount of training.
\end{abstract}

\section{Nomenclature}

CAS Calibrated airspeed

EAS Equivalent airspeed

EPR Engine pressure ratio

FL Flight level

GS Glide slope

HMI Human-machine interface

ILS Instrumented landing system

MSL Mean sea level

N1 Fan rotation speed

NM Nautical miles

PFD Primary flight display

RMSE Root mean square error

RNP Required navigation performance

SPL Spoiler

THR Throttle

TLX Task load index

WP Waypoint
Subscripts

com Command

err Error

$K \quad$ Inertia

$k \quad$ Flight path direction, or counting variable

$M D \quad$ Minimum drag

$S \quad$ Spoiler

$T \quad$ Thrust

tot Total

$W \quad$ Wind

$x \quad$ Longitudinal direction

$z \quad$ Normal direction

Symbols

$D \quad$ Drag force, $\mathrm{N}$

E Energy, J

$F \quad$ Thrust force, N, or transfer function

$g \quad$ Acceleration of gravity, $\mathrm{m} / \mathrm{s}^{2}$

$H \quad$ Altitude, $\mathrm{m}$

\footnotetext{
*Research Scientist, Flight Mechanics, Flight Control and Aeroelasticity, Marchstr. 12 - F5, 10587 Berlin, AIAA Member. ${ }^{\dagger}$ Research Scientist, Work, Engineering and Organizational Psychology, Marchstr. 12 - F7, 10587 Berlin.

¥Professor, Flight Mechanics, Flight Control and Aeroelasticity, Marchstr. 12 - F5, 10587 Berlin, AIAA Associate Fellow. §Professor, Work, Engineering and Organizational Psychology, Marchstr. 12 - F7, 10587 Berlin.
} 


$\begin{array}{ll}K & \text { Controller gains } \\ k & \text { Constant factor } \\ L & \text { Lift force, N } \\ L A & \text { Lever activity, } \% \\ L P & \text { Lever position, } 1 \\ L T & \text { Lever threshold, } 1 \\ N & \text { Sample size } \\ n & \text { Load factor, } 1, \text { or sample size } \\ p & \text { Probability value, } 1 \\ s & \text { Complex frequency, } 1 / \mathrm{s} \\ S D & \text { Standard deviation } \\ T & \text { Controller time constants, } \mathrm{s}\end{array}$

$\begin{array}{ll}t & \text { Time, } \mathrm{s} \\ V & \text { Speed, } \mathrm{m} / \mathrm{s} \\ W & \text { Weight, } \mathrm{N} \\ x & \text { Flight state parameter } \\ Z & \text { Trimmed flight state } \\ \alpha & \text { Angle of attack, deg, or significance level, } 1 \\ \delta & \text { Lever position } \\ \eta_{K} & \text { Flap deflection angle, deg } \\ \gamma & \text { Flight path angle, deg } \\ \gamma_{E} & \text { Total energy angle, deg } \\ \sigma & \text { Thrust incidence angle, deg } \\ \tau & \text { Time constant, } \mathrm{s}\end{array}$

\section{Introduction}

National and international institutions predict increasingly demanding requirements along with future growing air traffic, see, e.g., Flightpath 2050 by European Commission. ${ }^{1}$ Introducing complex flight trajectories optimize the use of airspace and decrease separation distances. The emerging precision requirements can be met for fully automated flight. However, it is essential that pilots can take manual control at any time, e.g., for short term flight path changes or in case of disabled auto pilot. Furthermore, the Federal Aviation Administration (FAA) recommends that pilots should sufficiently train manual flying skills. ${ }^{2}$ The expected high precision requirements of future air traffic will significantly raise the workload in manual flight.

Fly-by-wire technology has changed the conventional direct relation between control device and control surface deflections to the command and control of flight parameters, e.g., the $n_{z}$ control law in Airbus aircraft. ${ }^{3}$ The control laws change the flight dynamics and aircraft response behavior by augmenting stability and compensating disturbances in manual flight. This reduces workload even though pilots stay in the loop as "controller". Today, this augmented manual control is only used for attitude control with the aerodynamic control surfaces. Especially, engines and spoilers (in the case of speedbrakes) are used only conventionally in manual flight. Pilots need to control the energy state of the aircraft by changing thrust and spoiler lever positions, i.e., fan rotation speed or pressure ratio and spoiler deflection, and adjust the inputs according to deviations from the intended vertical flight path. The reactions to an input depend on the flight state. Additionally, as thrust and aerodynamic drag affect energy state, the pilots have to anticipate this by simultaneously monitoring speed and altitude changes. This highly complex control concept generates high cognitive and motoric workload that will increase with the future flight path precision requirements.

To address this problem, Schreiter et al. ${ }^{4}$ and Müller et al. ${ }^{5}$ introduced the flight control augmentation system nxControl. It provides a control law for longitudinal load factor $n_{x}$ by controlling engines and spoilers. To the best of our knowledge, the parameter $n_{x}$ is currently not used as a control variable for manual flight, neither in commercial airliners nor in research. The proposed human-machine interface (HMI) allows direct control and monitoring of the energy state rates as well as the system functions. The aim of the system is to enable manual flight under the demanding requirements of future air traffic. The system and the flight mechanical background were described by Müller et al. ${ }^{5}$ with focus on the HMI. The precursor evaluation study with short flight tasks showed that pilots were able to successfully fulfill the given tasks with satisfactory performance but lower thrust lever activity after only a short period of training with nxControl. The expected significant difference in precision compared to the conventional manual flight did not occur. One reason might be that the given tasks were easy to handle conventionally as well as with nxControl and therefore the benefits of the augmentation system could not emerge.

Meanwhile, the nxControl system has been improved with an optimized control law and a new inceptor. In the following sections the nxControl system is described with focus on design of the control laws. The flight mechanical background and the HMI are briefly recapitulated. Following this, a new evaluation study with 24 airline pilots is presented, showing the influences of the nxControl system for a demanding scenario. The pilots had to fly an approach pattern through the mountainous area of Salzburg airport under required navigation performance (RNP) conditions, with steep glide slope and wind disturbance. The results confirm the findings of the precursor study and show further positive effects of the nxControl system to precision and workload. 


\section{The Flight Control Augmentation System nxControl}

In today's sidestick controlled passenger aircraft, the vertical load factor $n_{z}$ is used to control pitching movements in manual flight. Together with thrust, pilots control airspeed and flight path angle. Engines are conventionally set either via fan rotation speed $N 1$ or engine pressure ratio (EPR). As an alternative, nxControl uses the longitudinal load factor $n_{x}$ for thrust control. With this value, pilots command the change of total energy (sum of potential and kinetic energy). Together with the $n_{z}$ command of the sidestick, pilots decide whether the energy change is converted to flight path angle and/or airspeed changes.

\section{II.A. Flight Mechanical Background}

The fundamental flight mechanical relationships are well established although under varying terminology (cf. Brockhaus $^{6}$ and Filippone ${ }^{7}$ ). The total longitudinal load factor is defined as the ratio of all external forces to weight. ${ }^{8}$ It corresponds to the total acceleration of the aircraft divided by the gravitational constant. The external forces can be calculated by the Newton's second law of motion for a rigid aircraft where all forces and the mass are concentrated at the center of gravity. Derived from the drag equation (longitudinal force equation) in flight path axes, the longitudinal load factor in flight path direction $n_{x k \text {,tot }}$ for symmetric flight (sideslip angle $\beta=0^{\circ}$, bank angle $\Phi=0^{\circ}$, change of azimuth angle $\dot{\chi}=0^{\circ} / \mathrm{s}$ ) is defined as follows:

$$
n_{x k, t o t}=\frac{1}{W}\left[F \cos \left(\alpha+\sigma-\alpha_{W}\right)-D \cos \alpha_{W}+L \sin \alpha_{W}\right]=\frac{\dot{V}_{K}}{g}+\sin \gamma .
$$

For a simplified description it is assumed that the thrust incidence angle $\sigma$ equals angle of attack $\alpha(\sigma=-\alpha)$ and that the wind angle of attack $\alpha_{W}$ is small. In horizontal flight, lift $L$ equals weight $W$ giving

$$
n_{x k, t o t}=\frac{F-D}{W}+\sin \alpha_{W}=\frac{\dot{V}_{K}}{g}+\sin \gamma .
$$

Firstly, Eq. (2) describes the dependence on the external influences of thrust force $F$ and aerodynamic drag force $D$, related to weight $W$ as well as the wind angle of attack $\alpha_{W}$. In addition, the correlation to the flight parameters longitudinal flight path acceleration $\dot{V}_{K}$, divided by the gravitational constant $g$, and flight path angle $\gamma$ are described. As the longitudinal load factor is proportional to the difference between thrust and drag for a constant wind, it is also known as specific excess thrust. Thrust and drag can be influenced actively by thrust input $\delta_{F}$, spoiler deflection $\delta_{S}$, and flap deflection $\eta_{K}$, whereas wind disturbs the longitudinal load factor(for brevity now referred to as $n_{x}$ ). However, pilots' inputs at the given control devices do not affect thrust and drag forces in a linear way. They are dependent on the actual flight state, i.e., airspeed and altitude.

Figure 1 shows thrust and drag force qualitatively as a function of equivalent airspeed $V_{E A S}$ and their dependencies on the control devices. If thrust equals drag, the speed remains constant (without wind influences) and corresponds to the intersection of the two graphs (trim point $Z_{1}$ ). With changing thrust

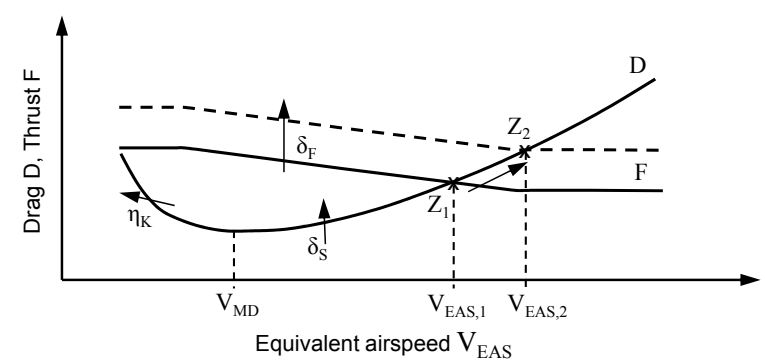

Figure 1. Thrust force $F$ and drag $D$ in dependence of input devices (thrust lever $\delta_{F}$, spoiler lever $\delta_{S}$, and flaps deflection $\eta_{K}$ ) as well as equivalent airspeed $V_{E A S}$; $Z_{1}$ and $Z_{2}$ mark trimmed flight states at $V_{E A S, 1}$ and $V_{E A S, 2}$ for two different thrust force $F$ via $\delta_{F}$ or drag force

$D$ via $\delta_{S}$ a difference appears, e.g., as shown by the shifted thrust curve (dashed line). Following Eq. (2), this difference can be either used to change airspeed or flight path angle, or both simultaneously. The first two cases are shown in figure 2. If the flight path is maintained (black dashed line), either manually or by an additional controller (e.g, $n_{z}$-controller), the excess thrust produces acceleration. With rising airspeed, drag increases (above minimum drag speed $V_{M D}$ ) and thus the acceleration decreases. After a comparatively long time (not shown in figure 2), the change in thrust or drag leads to a new trimmed state $Z_{2}$. If, on the other hand, the longitudinal load factor is used for changing altitude while speed stays constant (blue full line), the drag does not change and therefore the excess thrust remains constant, too. 
A preceding study ${ }^{9}$ showed, as expected, that pilots not only control speed but also altitude changes with thrust and spoiler settings. As described before, it would be enough to set these to specific positions and maintain altitude with elevator in order to change from state $Z_{1}$ to $Z_{2}$. To reach a higher acceleration, pilots further increase thrust, because otherwise the convergence to the new state would be too slow. The pilots therefore change the initial attitude
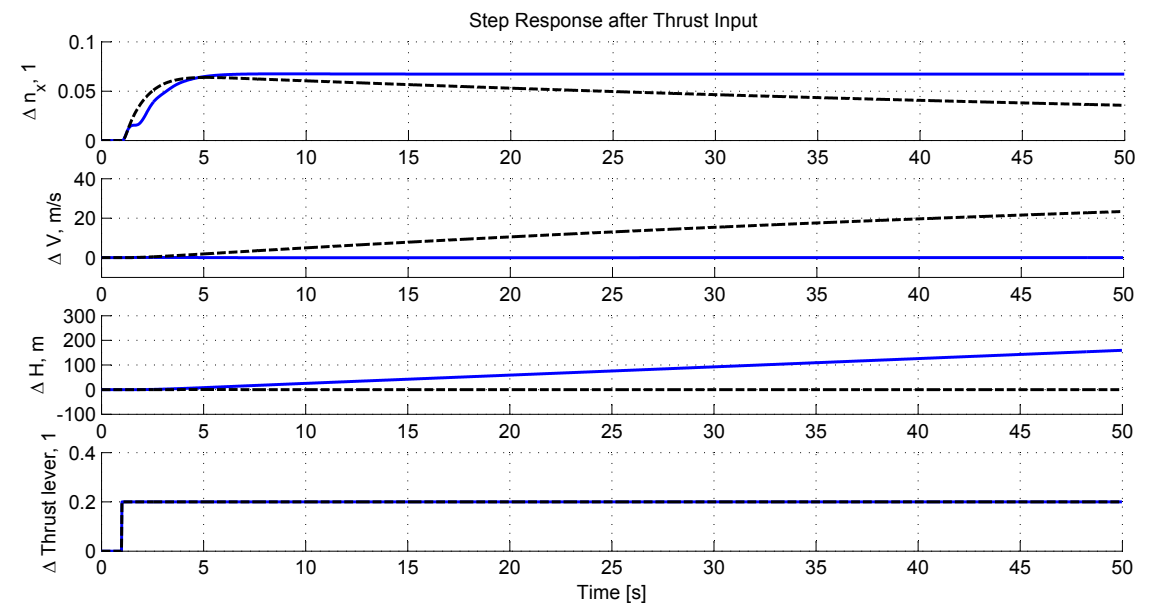

Figure 2. Step response of load factor $n_{x}$, airspeed $V$, and altitude $H$ to thrust lever input for speed hold (blue full line) and altitude hold (black dashed line).

command to rate command. In order to reach a different altitude at constant speed, pilots control climb rate with thrust and sink rate with thrust and spoilers. Speed is controlled indirectly by changing pitch attitude, see also Soule. ${ }^{10}$

The nonlinear relationships of aircraft motion depend on the actual flight state and are difficult to assess, especially due to the engines' delayed reaction. The typical pilot strategy is to use block inputs for thrust and spoiler levers. They command empirical values and adjust them if the expected changes in speed or flight path angle do not occur. "Pitch-and-power" tables with precalculated reference data for fan rotation speed and pitch angle for steady states at different altitudes and speeds are used as an aid. However, pilots can estimate the characteristics of the aircraft's reaction only qualitatively. Precise setting and maintaining the intended flight condition usually requires several adjustments.

These work sequences need to be improved in order to perform a more precise manual flight with lower workload. The nxControl system aims to support the manual flight by changing the input from the conventional parameters (N1 or EPR and incremental spoiler setting) to a meaningful flight parameter that better relates to the intended flight state changes. For a better interpretation of the load factor $n_{x}$ by the pilots, the relation of acceleration and flight path angle to the change in total energy is used. This is described by the total energy angle $\gamma_{E}$ (also called total flight-path climb angle ${ }^{8}$ )

$$
\sin \gamma_{E}=\frac{\dot{E}_{t o t}}{W V_{K}}=\frac{\dot{V}_{K}}{g}+\sin \gamma .
$$

The total energy angle $\gamma_{E}$ quantifies the change in total energy $\dot{E}_{t o t}$ related to weight and flight path velocity in an angular value and equals the load factor $n_{x}$ in Eq. (2). For manual flight, these equations describe the ability of the pilot to change the total energy state of the aircraft with a specific rate by setting thrust or drag. By changing the flight path angle, the pilot can distribute this total energy rate to potential and/or kinetic energy changes. The total energy angle has the same unit as the flight path angle and their relation can be directly compared. The difference provides the speed change information $\dot{V}_{K} / g$ (see Eq. (3)). Displaying the total energy angle instead of the load factor $n_{x}$ improves the human-machine interaction as pilots are familiar with the angular value of the flight path angle. Therefore, the pilots can better predict the change in flight state induced by thrust and spoiler commands. Together with the nxController described below, they can additionally command the intended change in flight state.

\section{II.B. Controller for the Longitudinal Load Factor}

The load factor $n_{x}$ is the command and control variable of the nxController. It is controlled by engines and spoilers. As two input devices are available for one control variable, a control allocation law is necessary. Methods for control allocation are described inter alia by Enns, ${ }^{11}$ Bodson ${ }^{12}$ or Oppenheimer. ${ }^{13}$ A preceding study $^{9,14}$ investigated how airline pilots use thrust and spoilers to manage energy state. It was found that pilots utilize thrust as primary control and spoilers as secondary control. They use spoilers additionally, if the energy decrease with idle thrust is not enough, and retract them before they raise thrust level. As pilot-centered design, the control allocation of nxControl is based on this behavior. Therefore, it consists of 
a logical switch toggling between two separate control laws for throttle and spoilers (see figure 3). One is active if spoilers are fully retracted and the other is active if engines are idle. For further enhancement of pilots' situation awareness a button on the HMI has to be pushed to allow the automated use of spoilers.

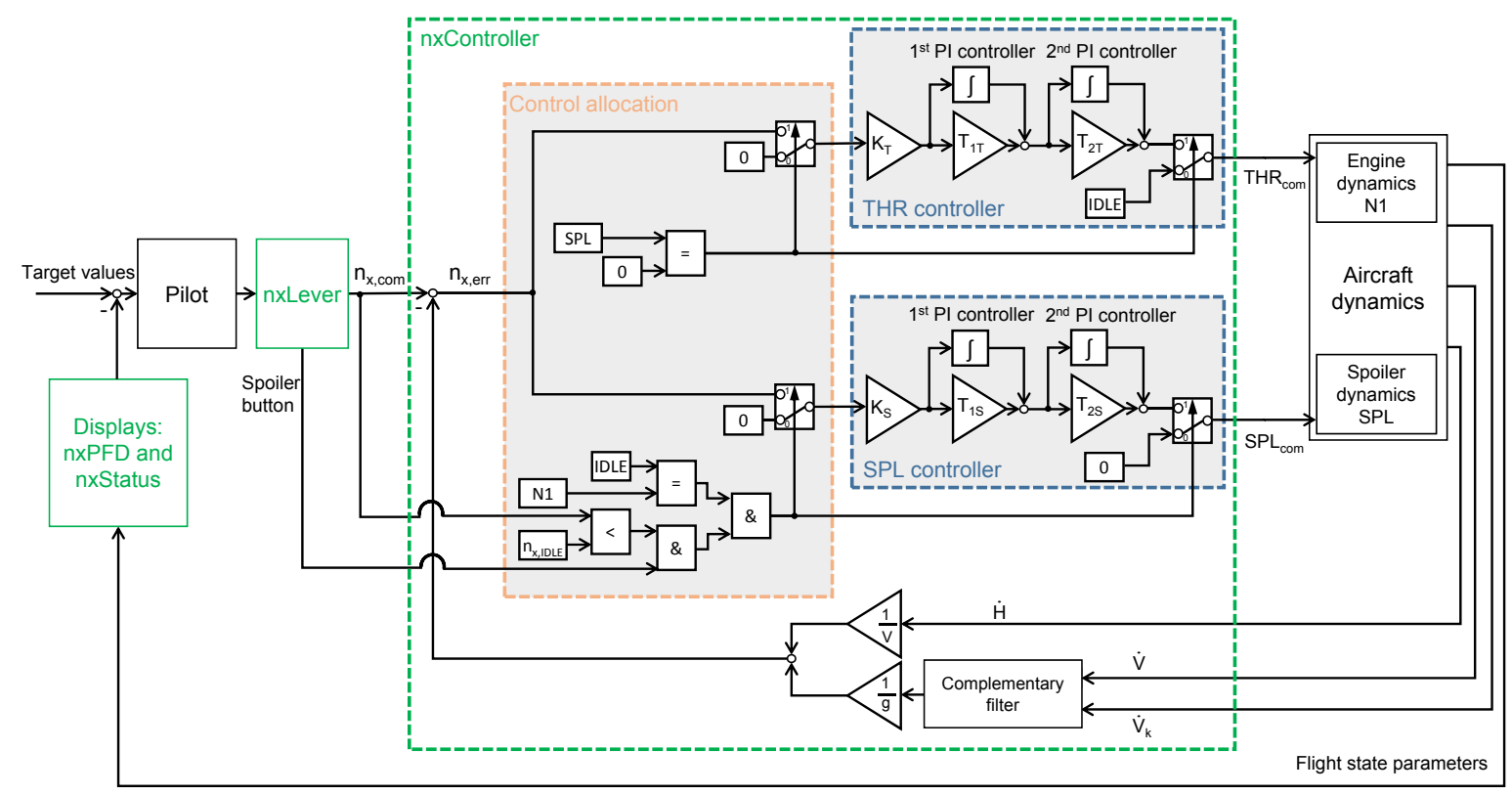

Figure 3. Control loop of the nxControl system with pilot in the loop.

The separation of the two control laws allows isolated controller designs for both inputs. To structure control laws architecture, linear model analysis was used for determining transfer functions of engine and spoiler inputs to load factor $n_{x}$ (resulting linear model of the uncontrolled system). For a steady airspeed, the responses can be approximated by aperiodic low-pass systems - for engines of third order and for spoilers of first order. However, at changing speed an additional term is necessary that describes the change of aerodynamic drag. The effects of speed variation to step response on $n_{x}$ was shown in figure 2. This influence can be approximated by a derivative element with first order lag (DT1). For each controller design, a distinguishing analysis of two modes - speed hold and altitude hold - is therefore necessary. The transfer function of thrust to load factor $F_{n_{x}, T H R}$ is given by

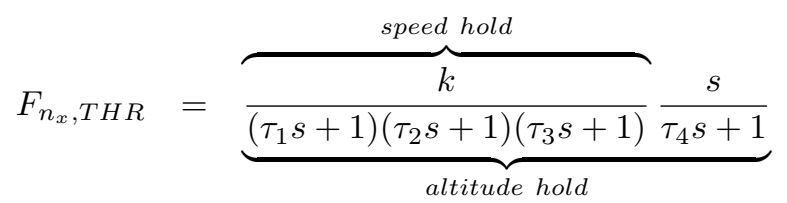

with the additional DT1 element in the altitude-hold mode. The transfer function of spoilers to load factor is derived in the same way.

These transfer functions, together with the following requirements, are the basis for architecture design of the controller in frequency domain. A primary requirement for the closed loop input response is steady-state accuracy, as pilots will not accept a system that does not realize their inputs. Therefore, the nxController needs an integrative behavior. As the altitude hold mode implements a zero to the open-loop system by the DT1 term, a further integrator eliminates this disturbance to steady-state accuracy. However, the use of integrators adversely affects dynamic stability, consequently the integrator effect is limited to low frequencies via two subsequent first-order proportional-integral controllers instead of two integrators. Figure 3 shows this architecture for both thrust and spoiler controller. The thrust controller transfer function (reaction of thrust command caused by error in load factor) $F_{T H R_{c o m}, n_{x, e r r}}$ is given with

$$
F_{T H R_{\text {com }}, n_{x, e r r}}=K_{T} \frac{T_{1 T} s+1}{s} \frac{T_{2 T} s+1}{s} .
$$

The transfer function of the spoiler controller $F_{S P L_{c o m}, n_{x, e r r}}$ has the same structure. 
As the altitude hold mode is more demanding in the case of steady-state accuracy, figure 4 shows the influence of the control architecture to the controlled step response to a $n_{x}$ command compared to the conventional thrust step input. The command value $\Delta n_{x, \text { com }}$ is 0.1 (in conventional case $\Delta N 1$ initially leading to $\left.\Delta n_{x, \text { com }}=0.1\right)$. It is apparent that the error in $n_{x}$ at rising speed for the conventional case is eliminated by increasing fan rotation speed by the controller. As a result, the speed acceleration is constant and allows for precise speed setting in relation to time constraints. The controlled step response for speed hold mode (not shown) is qualitatively similar to the conventional response as no thrust adjustments are necessary. However, with nxController the pilot does not have to search for the right fan rotation speed for the required flight path angle.

The controller gains were designed with the software tool MOPS (Multi-Objective Parameter Synthesis) by the German Aerospace Center. ${ }^{15}$ Both the described linear model and a highly sophisticated nonlinear simulation model were implemented in MOPS for optimization. A set of optimization parameters for time and frequency domain were set up as so called bad/good criteria that were acquired by the preliminary studies, standard controller requirements and model analysis. There are existing requirements and methods for longitudinal flying and handling qualities, e.g., $\mathrm{C}^{\star}$-criterion, ${ }^{16}$ Neal-Smith-criterion ${ }^{17}$ or Control Anticipation Parameter. ${ }^{18}$ However, most of them consider only the pitch dynamic above $0.05 \mathrm{~Hz}$. The flight path dynamic that is relevant for the nxControl system is below this frequency border. Therefore, these criteria could only inspire the requirements for the nxController design. At frequency domain, the damping ratio of control variable, the damping ratio of input variable, and the time delay margin were suitable criteria. In time domain, rise time, overshoot, steady-state offset, maximum error, and mean error of the control variable were used in both linear and nonlinear simulation. With these criteria, set in narrow limits, a schedule of gains depending on speed and altitude was obtained. Therefore, the flight envelope was discretized in a speed and altitude vector. At the center of the flight envelope, a set of gains was globally optimized. Starting with this gain set, neighboring flight states were then locally optimized. Therefore, a similar step response to the same input in different flight states is possible. Since control variable $n_{x}$ is derived by airspeed and flight path angle, every disturbance in energy state reaches the controller, e.g., changes in aircraft configuration or wind speed. In these situations, the pilot's workload can be reduced as readjustments are not necessary, once the right target value has been set. However, a complementary filter for airspeed and flight path speed reduces influences of turbulence on thrust control.

\section{II.C. HMI for Controller and Visualization of Energy Angle}

The human-machine interface comprises command input and monitoring devices for the load factor $n_{x}$. The load factor is represented by the total energy angle in degrees. This value can easily be connected to the pitch scale and the flight path angle indicator. Additionally, it provides general awareness of energy changes and distribution. Three new elements were added to the standard cockpit layout.

First, the information on the primary flight display (PFD) was extended by a symbol for the total energy angle (horizontal line) allowing tracing of energy changes (called nxPFD, see figure 5(a)). The "birdy" as common symbol for flight path vector was changed to a circle with center dot that indicates flight path angle without drift information. The flight path angle marks the change in altitude. The difference between energy and flight path angle represents the change in speed in a direct and centralized way. These symbols show the current state and are usable even without nxController. Similar concepts of implementing energy information to the cockpit have been introduced by inter alia Klopfstein, ${ }^{19}$ Lambregts et al. ${ }^{20}$ and Amelink et al., ${ }^{21}$ and are used in some head-up displays. ${ }^{22}$ 


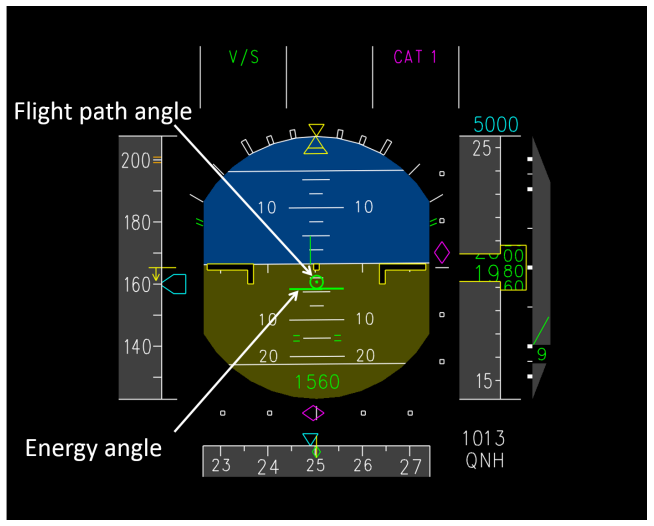

(a) nxPFD: Total energy angle and flight path angle at artificial horizon

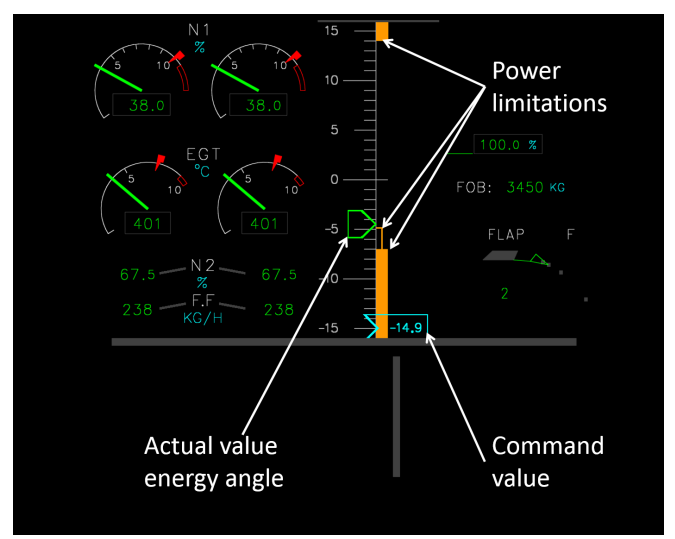

(b) nxStatus at the engine warning display: vertical degree scale for energy angle and command value

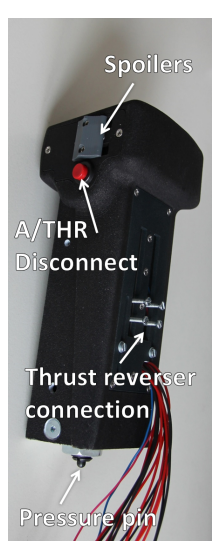

(c) nxLever: handle with spoiler switch

Figure 5. HMI of the nxControl system

The nxStatus display as visual interface to the nxController is placed on the Engine Warning Display (EWD) as shown in figure 5(b). On a vertical scale, analog to the pitch scale at the PFD, the commanded control value (blue flag), the actual value (green marker), as well as the upper and lower limits for engines thrust and spoilers are depicted. The limits represent the performance envelope (minimum and maximum energy rates) at a certain flight state. They change depending on air speed, altitude and aircraft configuration. Two lower limits are 1) the minimum energy angle at idle thrust (hollow lower strip) and 2) the minimum energy angle at idle thrust with spoilers fully extracted (filled lower strip). As the controller only uses the spoilers if the pilot activates them, the actual value of energy angle cannot fall below the first limit before the activation. Figure 5(b) shows a situation where the pilot commands a minimum value that lies below lower limits. The command value is at the lower end of the scale. The actual value marks the energy angle with idle thrust, because the pilot did not allow spoiler activation. Therefore the lower limit value of idle thrust is set as input. Another presentation of the performance envelope information was also proposed by Rijneveld ${ }^{23}$ using a Vertical Situation Display and performance information along vertical flight path. Concepts for scales representing thrust limits as well as command and actual value were patented by Artini ${ }^{24}$ and integrated in the PFD by Wyatt. ${ }^{25}$

The nxLever is the inceptor for the nxController. It is standard that one thrust lever is installed for each engine. As the nxLever issues one command value, only one lever is sufficient for all engines. Figure 5(c) shows a prototype of the handle with the common devices for auto thrust disconnect and thrust reverser as well as the sliding switch for activation of spoilers. If a command for energy change is below the realizable value at idle thrust this switch can be slid backwards. This allows the use of spoilers to manipulate the control value. The switch flips forward automatically if thrust was intermediately used for control. Then the activation has to be renewed. The lever movement is similar to the conventional thrust lever, but has a notch at the middle position. If the pressure pin on the handle bottom latches the notch, the command stands for maintaining the current total energy state. Above and below, energy changes are commanded linearly to the lever position.

\section{Evaluation Study}

The nxControl system was evaluated in a flight simulator campaign. The objective was to verify whether the assumptions regarding beneficial effects for precision and pilot workload could be met. In particular it was hypothesized, that

$\mathrm{H} \# 1 \mathrm{nxControl}$ allows a more precise control of longitudinal acceleration and with this a better tracking of the flight path, and

$\mathrm{H} \# 2 \mathrm{nxControl}$ relieves the pilots of frequent thrust adjustments and therefore lowers cognitive and motoric workload in manual flight compared with conventional manual flight. 
In previous investigations the nxControl system was tested initially with short flight tasks (airwork) ${ }^{5}$ and a standard straight in approach to runway $25 \mathrm{R}$ at Frankfurt (Main). ${ }^{26,27}$ It turned out that the pilots were able to fulfill the given tasks with nxControl. Moreover, they could perform the tasks with the same precision as with conventional thrust control even with short training. Even more important, they were able to achieve this precision with fewer movements of the thrust lever. A more challenging and demanding flight task was chosen for the present study. Pilots had to perform an approach to Salzburg with required navigation performance RNP 0.1. Such a complex task should work out the advantages of the nxControl system as compared to conventional pitch-and-power flying.

The experiments were conducted in the fixed-base research flight simulator SEPHIR (Simulator for Educational Projects and Highly Innovative Research) ${ }^{28}$ at the Chair of Flight Mechanics, Flight Control and Aeroelasticity of Technische Universität Berlin. The simulator cockpit is equipped with displays and sidestick (including control laws) similar to an Airbus aircraft.

A sample of 24 male certified pilots from commercial airlines with Airbus type ratings (A320: $N=20$, A330/A340: $N=3$, A380: $N=1$ ) participated in this experimental study. The 10 captains, 2 senior first officers and 12 first officers were aged between 24 and 63 years, with mean age of 40 years $(S D=12.6 a)$, and had an average flight time of 8505 hours $(S D=7422.4 \mathrm{~h}$, range $600 \mathrm{~h}$ to $25000 \mathrm{~h})$

\section{III.A. Flight Scenario and Procedure}

An existing RNP approach pattern to Salzburg SZG33 ${ }^{29}$ was used which is limited by terrain on both sides and, furthermore, has a steeper glide slope $\left(3.6^{\circ}\right)$ than usual approaches. The required performance of RNP 0.3 was aggravated for the study to RNP 0.1 in order to further increase the already high demands on energy management. The deviation to the target path was displayed using the available rhombuses and dots of the Instrumented Landing System (ILS) in the PFD. One dot lateral marked a deviation of $0.1 \mathrm{NM}$ and one dot vertical a deviation of $100 \mathrm{ft}$. The pilots were instructed to perform the flight as precisely as possible.

Figure 7(a) shows the vertical profile together with the points where aircraft configuration and speed had to be changed according to the lateral distance to the next waypoint. A flight procedure adapted to the experimental aim was provided that the pilots had to follow as accurately as possible. The procedure increased the requirements to manual flight even more, as the glide slope has to be intercepted with rather high speed in clean configuration at $12000 \mathrm{ft}$ above mean sea level (MSL). Additionally the stepwise configuration of the aircraft kept the workload of the pilots high until shortly before the end of the approach. The procedure started with a straight flight segment with speed and configuration changes up to waypoint WP2. A left turn to WP3 with configuration change followed and after a short straight middle section a second turn to the right with another configuration change was initiated at WP4. The last part until decision height at $2550 \mathrm{ft}$ MSL included a straight flight with several speed and configuration changes which was finished by a short right turn to runway direction at WP6.

In order to force the use of flight instruments (head down) the visibility range of the outside view was decreased by fog. The runway came in sight shortly before decision height. Additionally a steady wind of $15 \mathrm{kt}$ from $57^{\circ}$ without turbulence disturbed the flight. It changed from crosswind to tailwind at the middle section and therefore perturbed the energy management.

The given points for speed change had to be used as beginnings of deceleration phases and all given waypoints had to be used as fly-over instead of fly-by waypoints. For technical reasons, all the participants sat in the captain's seat, irrespective of their usual position.

\section{III.B. System Configurations and Measures}

The experiment was conducted with the three system configurations

- conventional (Conv.),

- energy display (nxPFD) with conventional thrust control, and

- nxControl (nxPFD, nxStatus and nxController).

The sequence of the system configurations was balanced across the pilots to control for effects due to fatigue or training. Furthermore, the procedure was repeated subsequently for each configuration to average the results of both trials.

To assess the effects of nxControl and nxPFD on flight path precision, the following flight parameters representing energy management were used for comparison: airspeed, altitude, flight path angle, and energy angle. 
As the procedure required certain flight path and speed targets, the measures for precision were given by the deviations of the flight parameters to their target values. Therefore, the root mean square error (RMSE) of the respective parameter $x$ at every time step $i$ (recorded with $50 \mathrm{~Hz}$ ) was used as defined by Eq. (6).

$$
\operatorname{RMSE}(x)=\sqrt{\frac{\sum_{i=1}^{n}\left(x_{i}-x_{\text {target }, i}\right)^{2}}{n}}
$$

The effect on workload was investigated with subjective questionnaires and objective measurements of lever movements. After each landing, the participants had to rate their subjective workload on the subscales of the NASA Task Load Index (TLX): mental demand, physical demand, temporal demand, performance, effort, and frustration. ${ }^{30}$ The over-all workload score was achieved without weighting the scales against each other as this was found to have a negligible effect on the results. ${ }^{31}$

In order to assess how much effort the pilots had to invest in thrust control, thrust lever movements were recorded throughout the flight simulation. A lever movement was defined as a change in lever position $L P$ higher than a threshold $L T=0.5 \%$ of the whole lever range $(0.2 \mathrm{~cm}$ at the lever top) in a time interval of $\Delta t=2 \mathrm{~s}$ (see Eq. (7)). The lever activity $L A$ was defined as the sum of these lever movements divided by the time samples $N=t / \Delta t$ (see Eq. (8)).

$$
\begin{aligned}
\text { count }_{k} & = \begin{cases}0, & \text { if }\left|L P_{t_{k}-\Delta t}-L P_{t_{k}}\right|<L T \\
1, & \text { if }\left|L P_{t_{k}-\Delta t}-L P_{t_{k}}\right| \geq L T\end{cases} \\
L A & =\frac{\sum_{k=1}^{N} \text { count }_{k}}{N}
\end{aligned}
$$

Lever activity $L A$ directly reflects the cognitive and physical workload related to thrust control. It is assumed that the higher this ratio (and therefore the more corrections of the input made) the higher is the workload for the given flight task.

\section{Results}

The results for the overall flight are shown as boxplots in figure 6. One box shows the $25 \%$ and $75 \%$ percentiles and the median. In addition, the mean value is shown as an asterisk. The data of the system configurations were analyzed pairwise in t-tests and multiple testing corrections according to Sidak ${ }^{32}$ were applied. This resulted in corresponding $p$-values (probability of random data distributions), which are depicted in the diagram titles. If the $p$-value is lower than the common significance level $\alpha=0.05$, the statistical significance of the change in mean value can be corroborated.

Furthermore, the time histories of precision and workload parameters were compared to give a more detailed view of the effect of the different system configurations. Therefore, the median of all pilots' progress data were taken per system configuration and are plotted against the distance to runway in figure 7 . The interval contains the beginning of the scenario at $32 \mathrm{NM}$ to the decision height at $3 \mathrm{NM}$ and is synchronized to the flight procedure.

\section{IV.A. Flight Precision}

Figures 6(a) to 6(d) show the root mean square errors (RMSE) of the chosen precision parameters as boxplots. As expected, the RMSE of airspeed was significantly lower when flying with nxPFD and nxControl as compared to the conventional configuration. However, a comparable effect on the RMSE of altitude was not found. Results for the two additional indicators in the nxPFD - flight path and energy angle - revealed differing effects. While the RMSE for the flight path angle only benefited from providing these indicators in the nxPFD configuration, a significantly lower RMSE for the energy angle was only found in the nxControl configuration.

The median time histories in figures 7(b) to 7(e) show the same precision parameters. The speed progress is depicted as speed deviation from the target speed (figure 7(b)), which is why the plot leaps at the beginning of speed change and gradually decreases afterward. Analogous to the statistical analysis of the RMSE, the time history shows a higher speed deviation in the conventional configuration compared to nxPFD and nxControl at several segments. First of all, it becomes obvious that the tolerance of $\pm 5 \mathrm{kt}$ was exceeded twice in the conventional case - after glide slope (GS) intercept and after flaps configuration to full (F4). 
These effects were eliminated by nxPFD, and nxControl lowers the deviation at these segments even more. At the ends of the speed change segments, it can be seen that steady flight is reached faster with nxControl, compared to both other configurations. However, slight constant speed deviations are observable (especially at the second speed reduction), which explains the equal RMSE compared to nxPFD. Energy disturbances, like the change in wind direction between WP3 and WP4 as well as flap configuration F3 and F4, have less influenced the speed deviation in nxControl than in nxPFD or the conventional configuration.

The altitude deviation, shown in figure 7 (c), confirms the statistical comparison. There are no evident differences that could be related to the given system configurations.

The time history of flight path angle (see $7(\mathrm{~d})$ ) also shows few differences between the system configurations. At GS intercept, the pilots reached smaller deviations to the target value with nxPFD and nxControl than in the conventional configuration. Additionally, at the second speed change (at approx. 23 to $22 \mathrm{NM}$ ) and third speed change (at approx. 12 to $10.5 \mathrm{NM}$ ), lower deviations from the target value are visible in the two nx configurations. However, at the turn between WP2 and WP3, nxControl shows the largest deviations of all system configurations. It is assumed that at this point the temporal coordination of the different tasks, deceleration with spoilers, flap configuration to F2, and initiation of the turn combined with the use of a new control system, was highly demanding and requires more training. Nevertheless, the following less demanding segments again show a lower deviation for the $\mathrm{nx}$ configurations.

In contrast, the time history of energy angle in figure $7(\mathrm{e})$ shows obvious differences between the system configurations. Overall, lower variance in progression can be identified for nxControl, caused by the controller automatically compensating for energy disturbances. This is most obvious when the flap configuration is changed to F1, F3, and F4. The nxPFD configuration only lowers the deviation at flap configuration F4. Additionally, the GS intercept was proceeded faster and more directly in nxControl than in both other system configurations, because commanding the target value is directly possible. The disturbance by wind change had only a slight effect on the energy angle in all configurations, although the most constant trend is visible at the nxControl configuration.

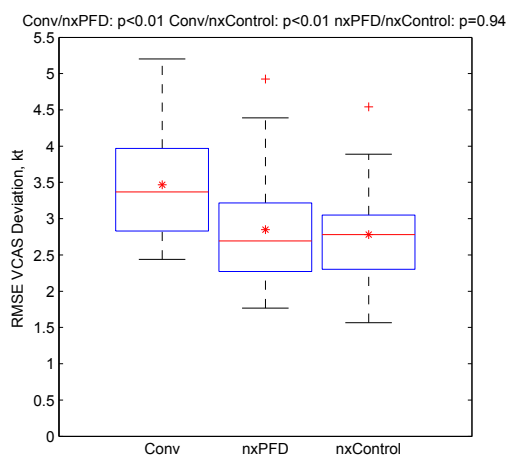

(a) RMSE of speed

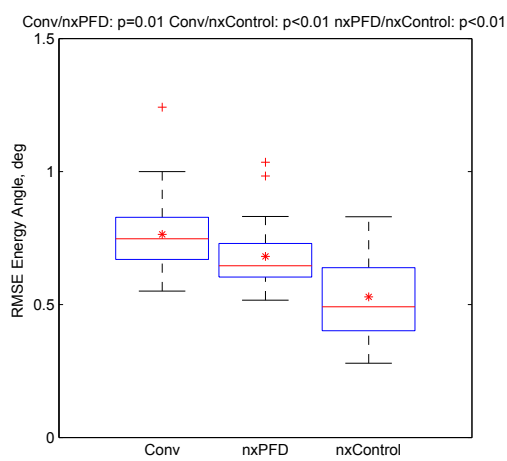

(d) RMSE of energy angle

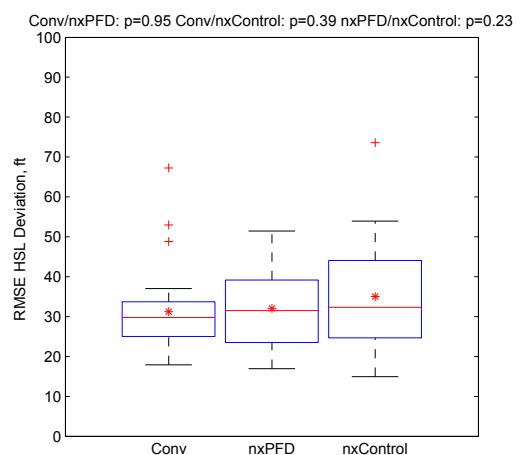

(b) RMSE of altitude

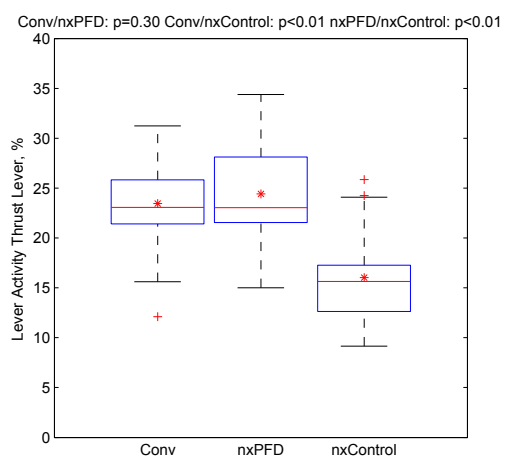

(e) Lever activity

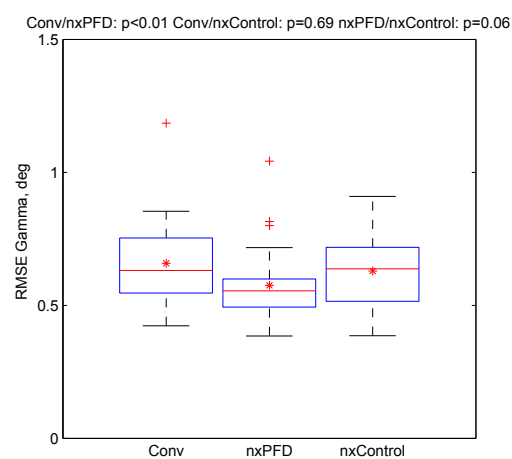

(c) RMSE of flight path angle

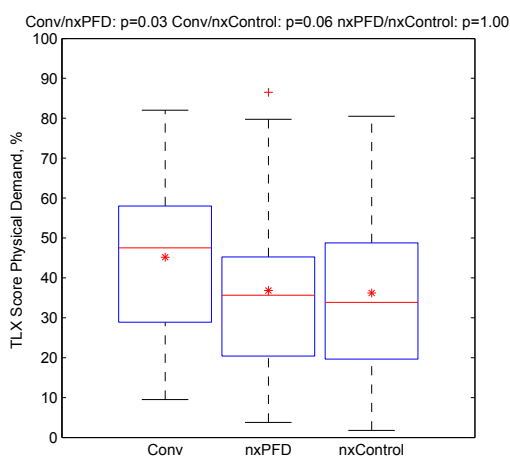

(f) TLX score physical demand

Figure 6. Statistical analysis via boxplots and means: RMSE values of precision and workload parameters. 


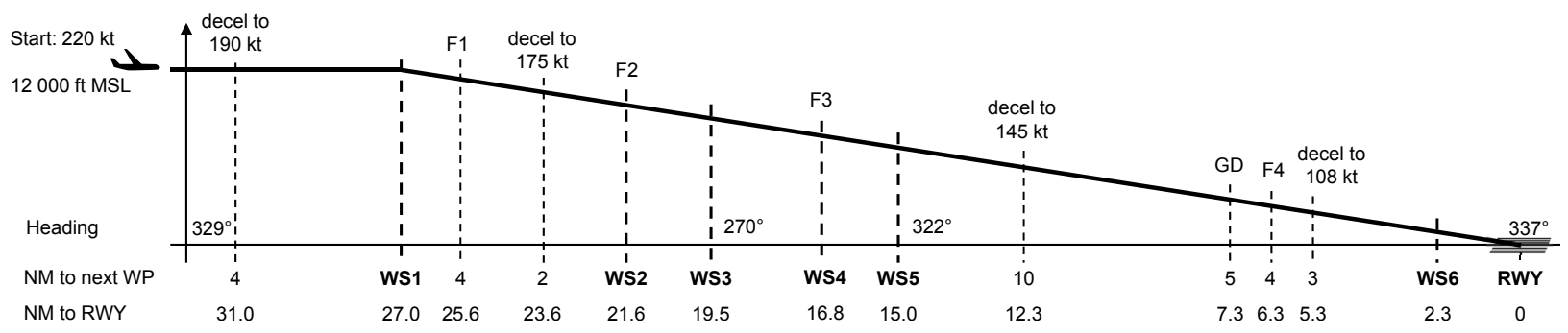

(a) Procedure on vertical path to runway

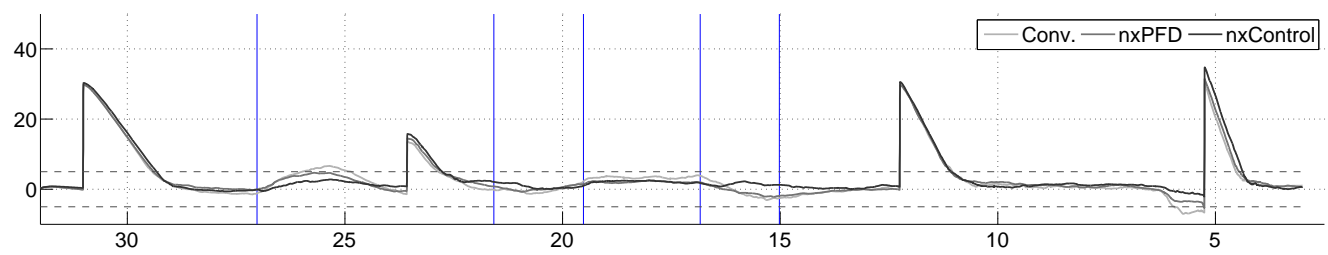

(b) Speed deviation in knots against distance to runway in NM

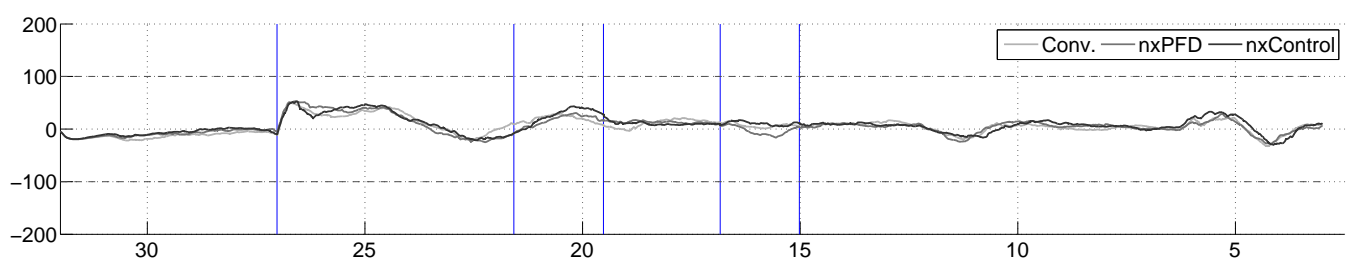

(c) Altitude deviation in feet against distance to runway in NM

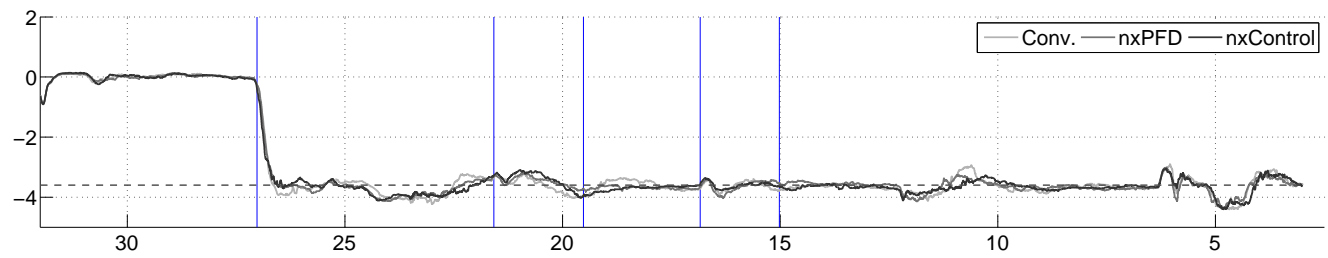

(d) Flight path angle in degrees against distance to runway in NM

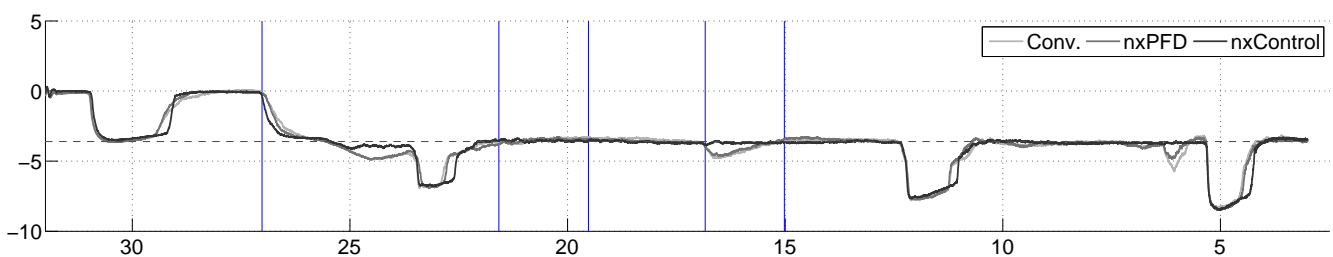

(e) Energy angle in degrees along against to runway in NM

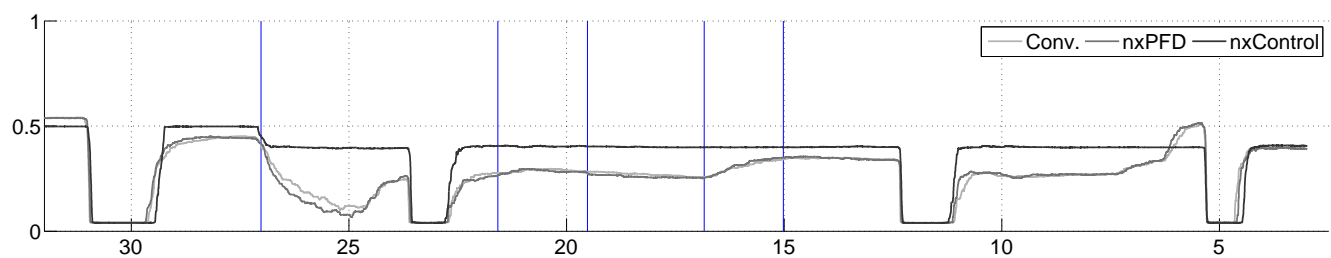

(f) Normalized thrust lever position against distance to runway in NM

Figure 7. Median histories of flight parameters averaging all participants per system configuration. Figure (a) shows the nominal vertical trajectory with waypoints and configuration changes. 


\section{IV.B. Workload}

The lever activity as a workload measure is shown in figure 6(e). As expected, the lever activity decreases significantly with nxControl in contrast to the conventional and nxPFD configurations. Only displaying energy information on the PFD is not enough to reduce physical workload. Additionally, a separate use of spoiler lever is not necessary with nxControl, which is not included to the statistics in figure 6(e). But, the subjective TLX questionnaire shows a slightly different picture. While the overall TLX score does not change significantly between the three system configurations (not illustrated), the sub scale of physical demand identifies a lower value for nxPFD and nxControl, compared to the conventional configuration (see fig 6(f)). Subjectively, the control of thrust seems to be more goal-oriented with nxPFD, but the introduction of the nxController did not lead to further benefits.

In figure $7(\mathrm{f})$ the time history of lever positions is shown. Note that with nxControl the positions mean different commands to the engines than in the conventional configurations. In a qualitative examination it becomes clear that pilots make fewer movements with nxLever to achieve the required precision. Especially at the segments of energetic disturbances, where the controller automatically compensates, no pilot input was necessary because nxControl laws keep the energy angle constant. This becomes especially apparent when flaps are extended and the wind changes (WP3 to WP4). In contrast, either in nxPFD or in the conventional configuration the pilots needed to tune the thrust setting manually and "search" for the right setting with small lever position changes. Clear differences also emerged in case of necessary speed reductions. In order to adjust the new target speed, faster, less, and more direct inputs could be made with the nxLever, while multiple incremental step inputs were required by conventional thrust control until the best position is found. Also at GS intercept, a defined lever movement was recognizable for nxControl, whereas for nxPFD and conventional configuration the multiple incremental search for the correct lever position can be observed.

\section{IV.C. Discussion}

The results show positive effects of the nxControl system with respect to a more precise control of flight path and airspeed during the approach. However, the precision in altitude remained comparable to that achieved with conventional thrust control. Most likely this is due to the aircraft behavior with flight path stable control laws via side stick. With this, changes in energy will be transferred to speed changes to maintain flight path in all cases (except speed protections). The nxControl system does not influence this behavior. This could be the reason why no differences in altitude and flight path angle were observable. Since all energy changes affect mainly the speed precision, the effects of nxPFD and nxControl can be observed in speed and energy parameters. Obviously, the centralized information on the nxPFD makes it possible to capture unintended changes in speed faster and more precisely than in the conventional case. This is due to the delayed reaction of the speed trend vector at the airspeed indicator that shows changes only over a certain threshold. This shows that the additional energy information leads to faster recognition of disturbances and pilots can react more quickly to maintain the energy state with the nxControl system. Furthermore, the nxController prevents errors in energy state by the given control law. Hypothesis $\mathrm{H} \# 1$ is therefore supported.

A more complex picture emerged for workload results. As expected, the objective parameter of lever activity was decreased significantly with nxControl. However, on the subjective level, a lower physical demand was also perceived in the condition nxPFD, which actually did not change anything with respect to control movements compared to conventional flying. The reason for this subjective perception is not clear. It can be assumed that this was caused by the pilots ability to find the correct thrust setting more targeted with the shown energy angle in the nxPFD in contrast to the "blind searching" for the right fan rotation speed in the conventional case.

The overall NASA TLX scores and all sub scales (except physical demand) did not show a significant effect compared to conventional control and PFD, neither for condition nxPFD nor nxControl. That is, the subjectively perceived overall workload did not differ between the three conditions. Considering the confrontation with a fully new and unknown control system and the rather short time for training, a higher workload compared to the well known and routinely used conventional system is not surprising. Additionally, the results could be affected by the unusual scanning pattern or motoric behavior of pilots who usually operate from the right hand cockpit seat. Given this, the fact that the new system obviously had not elevated the load, might already be taken as evidence that the nxControl concept can be easily learned and understood. Thus, hypothesis H\#2 can be confirmed for physical workload. For cognitive workload, it initially has to be rejected, but it might be supported with more training with nxControl. 


\section{Conclusion}

The nxControl system aims to enable a more precise manual flight on highly demanding trajectories at lower workload compared to today's manual flight. For this purpose, the system and evaluated in a flight simulator campaign with airline pilots. The overall goal was achieved, as smaller mean errors were made in maintaining precision parameters airspeed and energy angle, in combination with lower physical activity with the nxControl system.

The motivations for the system are the rising requirements of future air traffic as well as safety aspects caused by loss of flight skills due to excessive autopilot deployment. Therefore, the system should increase safety addressing

- continuous training of flying skills by more manual flight in daily operations,

- minor changes in workflow due to nxControl in comparison to conventional flight to avoid influences to the basic flight skills, and

- advanced awareness and control of energy state.

The results of this study provide evidence for a more precise manual flight at reduced workload using the nxControl system in highly demanding situations. Therefore, pilots could be able to fulfill the requirements of the future air traffic with lower workload in manual flight. It can be assumed that this will allow for more frequent manual flight. The lever movements with nxControl are similar to those of a conventional thrust lever. Thus, the basic mode of operation is only marginally affected. However, by automatically compensating for energy disturbances, such as wind or flap settings, the standard control strategy changes with the nxControl system. Additionally, the scanning pattern changed from basic parameters like pitch and fan rotation speed to physical flight path parameters like flight path angle and energy angle. How these changes in workflow affect the flight skills should be scrutinized critically, especially in failure conditions of the nxControl system. Beyond this worst case scenario, the validated additional information on the nxPFD about development and distribution of energy and the direct control of energy change with nxControl enables a targeted and precise manual control for future air traffic. Although not all working hypotheses could be supported, the overall pattern of results provides evidence for the potential of nxControl to enhance both the precision and safety of manual flight.

\section{Acknowledgments}

This work is funded by the DFG (German Research Foundation) under contract LU 1397/3-1, MA 3759/3-1. The authors thank the pilots who participated in the described experiments as well as B. Boche, D. Bieniek, A. Kaden, I. Karakaya, and M. Schaumburg for their support.

\section{References}

${ }^{1}$ EC (European Commission), "Flightpath 2050, Europe's vision for aviation," Report of the High Level Group on Aviation Research, 2011.

${ }^{2}$ FAA (Federal Aviation Administration), "Safety Alert for Operators, Manual Flight Operations, SAFO 13002," http://www.faa.gov/ other_visit/ aviation_industry/ airline_operators/ airline_safety/ safo/ all_safos/ media/ 2013/ SAFO13002.pdf, 2013.

${ }^{3}$ Favre, C., "Fly-by-wire for commercial aircraft: the Airbus experience," International Journal of Control, 1994, pp. 139157.

${ }^{4}$ Schreiter, K., Müller, S., and Luckner, R., "nxControl: Konzept zur Vorgaberegelung für die Längsbeschleunigung des Flugzeugs," Deutscher Luft- und Raumfahrtkongress 2013, Stuttgart, 2013, urn:nbn:de:101:1-2013112216214.

${ }^{5}$ Müller, S., Schreiter, K., Manzey, D., and Luckner, R., "nxControl instead of pitch-and-power: A Concept for Enhanced Manual Flight Control," CEAS Aeronautical Journal, 2016, pp. 107-119, DOI 10.1007/s13272-015-0169-9.

${ }^{6}$ Brockhaus, R., Alles, W., and Luckner, R., Flugregelung, Springer Berlin Heidelberg, 3rd ed., 2011.

${ }^{7}$ Filippone, A., Advanced Aircraft Flight Performance, Cambridge University Press, 2012.

${ }^{8}$ International Standard Organisation (ISO), "Flight dynamics - Concepts, quantities and symbols - Part 1: Aircraft motion relative to the air, ISO1151-1," 1988.

${ }^{9}$ Müller, S., Schreiter, K., and Manzey, D., "Simulatorstudie zur Ermittlung mentaler Handlungsmodelle von Piloten beim Energiemanagement," Deutscher Luft- und Raumfahrtkongress 2013, Stuttgart, 2013, urn:nbn:de:101:1-2013102515192.

${ }^{10}$ Soulé, H. A., "The Throttle Controls Speed, Right? Wrong!" Astronautics and Aeronautics, 1969.

${ }^{11}$ Enns, D., "Control Allocation Approaches," Proceedings of the AIAA GNC Conference, 1998, pp. 98-108.

${ }^{12}$ Bodson, M., "Evaluation of Optimization Methods for Control Allocation," Journal of Guidance, Control, and Dynamics, 2002, pp. 703-711. 
${ }^{13}$ Oppenheimer, M. W., Doman, D. B., and Bolender, M. A., "Control Allocation for Over-actuated Systems," 14 th Mediterranean Conference on Control and Automation, 2006.

${ }^{14}$ Müller, S., Manzey, D., Bleyer, A., Schreiter, K., Voigt, A., and Luckner, R., "Untersuchung der mentalen Repräsentation von Energiemanagement bei der Flugzeugführung zur Entwicklung eines Pilotenassistenzsystems," Grundlagen und Anwendungen der Mensch-Maschine-Interaktion: 10. Berliner Werkstatt - Mensch-Maschine-Systeme, 2013, pp. 230-237, urn:nbn:de:kobv:83-opus4-42897.

${ }^{15}$ Joos, H., Bals, J., Looye, G., Schnepper, K., and Varga, A., "A multi-objective optimisation-based software environment for control systems design," IEEE International Symposium on Computer Aided Control System Design, 2002, pp. 7 - 14.

${ }^{16}$ Tobie, H., Elliot, E., and Malcom, L., "A new longitudinal handling qualities criterion," Proceedings of the National Aerospace Electronics Conference, 1966, pp. 93-99.

${ }^{17}$ Neal, T. and Smith, R., "An In-Flight Investigation to Develop Control System Design Criteria for Fighter Airplanes," Air Force Flight Dynamics Lab., AFFDL-TR-70-7, 1970.

${ }^{18}$ Bischoff, D., "The Control Anticipation Parameter for Augmented Aircraft," Naval Air Development Center Warminster PA, Aircraft and Crew Systems Technology Directorate, 1981.

${ }^{19}$ Klopfstein, G., "Rational Study of Aircraft Piloting," Advanced Aircrew Display Symposium, 1976, pp. 231-248.

${ }^{20}$ Lambregts, T., Rademaker, R., and Theunissen, E., "A new ecological primary flight display concept," 2008 IEEE/AIAA 27th Digital Avionics Systems Conference, 2008.

${ }^{21}$ Amelink, M. H., Mulder, M., Paassen, V., and Flach, J., "Theoretical Foundations for a Total Energy-Based Perspective Flight-Path Display," International Journal of Aviation Psychology, Vol. 15, No. 3, 2005, pp. 205-231.

${ }^{22}$ Blaye, P. L., Roumes, C., Fornette, M.-P., and Valot, C., "Head Up Displays symbology (HUD): Pre normative study for DGAC/SFACT." Tech. Rep. June, Onera, Paris, 2002.

${ }^{23}$ Rijneveld, P., Borst, C., Mulder, M., and van Paassen, M. M., "Towards Integrating Traffic and Terrain Constraints," AIAA Guidance, Navigation, and Control Conference, 2010, pp. 1-20.

${ }^{24}$ Artini, F., Demortier, J.-P., Bouchet, C., and Airbus France, "Flight Control Indicator Displaying the Aircraft's Thrust Information," U.S. Patent, US 7636617 B2, 2009.

${ }^{25}$ Wyatt, I. S. and Honeywell International Inc., "Aircraft Display With Potential Thrust Indicator," U.S. Patent, US $6262674 \mathrm{~B} 1,2001$.

${ }^{26}$ Müller, S., Manzey, D., Schreiter, K., and Luckner, R., "Implementing Energy Status in Head-Down Cockpit Displays: Impact of Augmented Energy Information on Pilot's Performance," 59th Annual Meeting of the Human Factors and Ergonomics Society, Human Factor and Ergonomics Society, Santa Monica, CA, 2015, pp. 926-930, DOI 10.1177/1541931215591270.

${ }^{27}$ Schreiter, K., Müller, S., Luckner, R., and Manzey, D., "Verbesserung von Flugpräzision und Arbeitsbeanspruchung bei manuellen RNP-Anflügen durch Vorgaberegler und Anzeigen für den Energiewinkel (nxControl)," Deutscher Luft- und Raumfahrtkongress 2016, Braunschweig, 2016, urn:nbn:de:101:1-201610283560.

${ }^{28}$ TU Berlin, Department of Flight Mechanics, Control and Aeroelasticity, "SEPHIR - Simulator for Educational Projects and Highly Innovative Research," http://www.fmra.tu-berlin.de/ menue/ forschung/ ausstattung/ sephir/.

${ }^{29}$ Austro Control GmbH, "Instrument Approach Procedure Salzburg RNAV (RNP) Z RWY 33, OWS AD 2.24-6-6," http:// eaip.austrocontrol.at/ lo/ 150430/ Charts/ LOWS/ LO_AD_2_LOWS_24-6-6_en.pdf, [cited 05.10.2015].

${ }^{30}$ Hart, S. and Staveland, L., "Development of NASA-TLX (Task Load Index): Results of empirical and theoretical research," Human mental workload, 1988, pp. 139-183.

${ }^{31}$ Byers, J. C., Bittner, A. C., and Hill, S. G., "Traditional and raw task load index (TLX) correlations: Are paired comparisons necessary?" Advances in Industrial Ergonomics and Safety, 1989, pp. 481-485.

${ }^{32}$ Wright, S. P., "Adjusted P-Values for Simultaneous Inference," BIOMETRICS, Vol. 48, 1992, pp. 1005 - 1013. 